\title{
Preoperative classification of submucosal fibrosis in colorectal laterally spreading tumors by endoscopic ultrasonography
}

Authors

Institutions
Tomoaki Makino', Shuji Kanmura', Fumisato Sasaki ${ }^{1}$, Yuichirou Nasu' ${ }^{1}$, Keita Funakawa ${ }^{2}$, Akihito Tanaka', Shiho Arima ${ }^{1}$, Junichi Nakazawa ${ }^{1}$, Hiroki Taguchi ${ }^{1}$, Shinichi Hashimoto ${ }^{1}$, Masatsugu Numata ${ }^{1}$, Hirofumi Uto ${ }^{1}$, Hirohito Tsubouchi ${ }^{2}$, Akio Ido ${ }^{1}$

${ }^{1}$ Digestive and Lifestyle Diseases, Kagoshima University Graduate School of Medical and Dental Sciences, Kagoshima, Japan ${ }^{2}$ Kagoshima City Hospital, Kagoshima, Japan submitted 13. May 2014 accepted after revision 16. January 2015

\section{Bibliography}

Dol http://dx.doi.org/ 10.1055/s-0034-1391782

Published online: 10.4.2015 Endosc Int Open 2015; 03: E363-E367

(C) Georg Thieme Verlag KG Stuttgart . New York E-ISSN 2196-9736

\section{Corresponding author}

\section{Shuji Kanmura}

Digestive and Lifestyle Diseases Kagoshima University Graduate School of Medical and Dental Sciences

8-35-1

Sakuragaoka

Kagoshima

890-8520 Japan

Fax: +81-99-2643504

skanmura@m2.kufm.

kagoshima-u.ac.jp
Background and study aims: Although endoscopic submucosal dissection (ESD) is an established therapy for colon neoplasms including laterally spreading tumors (LSTs), its application to advanced fibrotic lesions is very difficult owing to the thin walls of the large intestine. We examined the ability of preoperative endoscopic ultrasonography (EUS) to predict lesion fibrosis in patients undergoing colorectal ESD.

Patients and methods: From 2009 to 2013, 58 LSTs were evaluated retrospectively with EUS and treated using colorectal ESD. The degree of submucosal fibrosis was determined during ESD

\section{Introduction}

\section{$\nabla$}

Although colorectal cancer (CRC) is the third most common malignancy in the world [1], early stage CRC is easily detected with the latest endoscopic innovations. Early CRC and slightly invasive submucosal CRC (invasion depth $<1000 \mu \mathrm{m}$ ) are indications for endoscopic resection [2,3]. In recent years, endoscopic submucosal dissection (ESD) has been commonly used in early stage CRC in Japan and other countries. In addition, colorectal ESD for colorectal laterally spreading tumors (LSTs) is a feasible technique for en bloc resection and was shown to result in no local recurrence [4]. However, ESD is technically difficult and results in frequent complications compared with conventional endoscopic mucosal resection (EMR) [5]. Technical challenges include factors related to tumor location, tumor size, and the presence of ulcer scars. Colorectal ESD is specifically complicated by the thinness of the intestinal wall and by lesions with severe fibrosis [6]. Although cancer in situ accompanied by submucosal fibrosis is one of the indications for ESD, severe submucosal fibrosis can lead to long treatment duration and unexpected complications such as perforation $[7,8]$. Thus, by anticipating the degree of fibrosis before colorectal ESD, it and classified as F0 (no fibrosis), F1 (mild fibrosis), or F2 (severe fibrosis).

Results: The sensitivity and specificity of fibrosis prediction by preoperative EUS of all cases were $77.8 \%$ and $57.1 \%$, respectively. However, there was a high accuracy $(97.2 \%, 35 / 36)$ for only the 36 LSTs with clear and visible images. In one case, EUS diagnosed no fibrosis but significant fibrosis was found during ESD, the result of colon cancer invasion into the submucosa.

Conclusions: Preoperative EUS before colorectal ESD successfully predicted the degree of fibrosis in a number of cases.

may be possible to select the operator according to the skill required for a given procedure.

Endoscopic ultrasound sonography (EUS) is useful for evaluating CRC invasion depth [9-14]. For instance, CRC described on EUS as a hypoechoic mass within the submucosal layer indicates deep submucosal invasion. Previous studies have shown that the accuracy of EUS for determining CRC invasion depth is $66-88 \%$ [9,15-17]. EUS is also useful in diagnosing depth of invasion of gastric cancer with or without peptic ulcer scar [17]. However, it remains unclear whether this technique can predict the presence of fibrotic lesions in the colon. In this study at our hospital, we examined the association between colorectal LST fibrosis predicted by EUS before colorectal ESD and the degree of submucosal fibrosis determined during ESD.

\section{Materials and methods \\ $\nabla$}

Patients

Between February 2009 and February 2013, 58 LSTs in 58 patients were evaluated with preoperative EUS and treated with colorectal ESD. LSTs were macroscopically classified as four subtypes. LSTs were first classified as granular type (LST- 


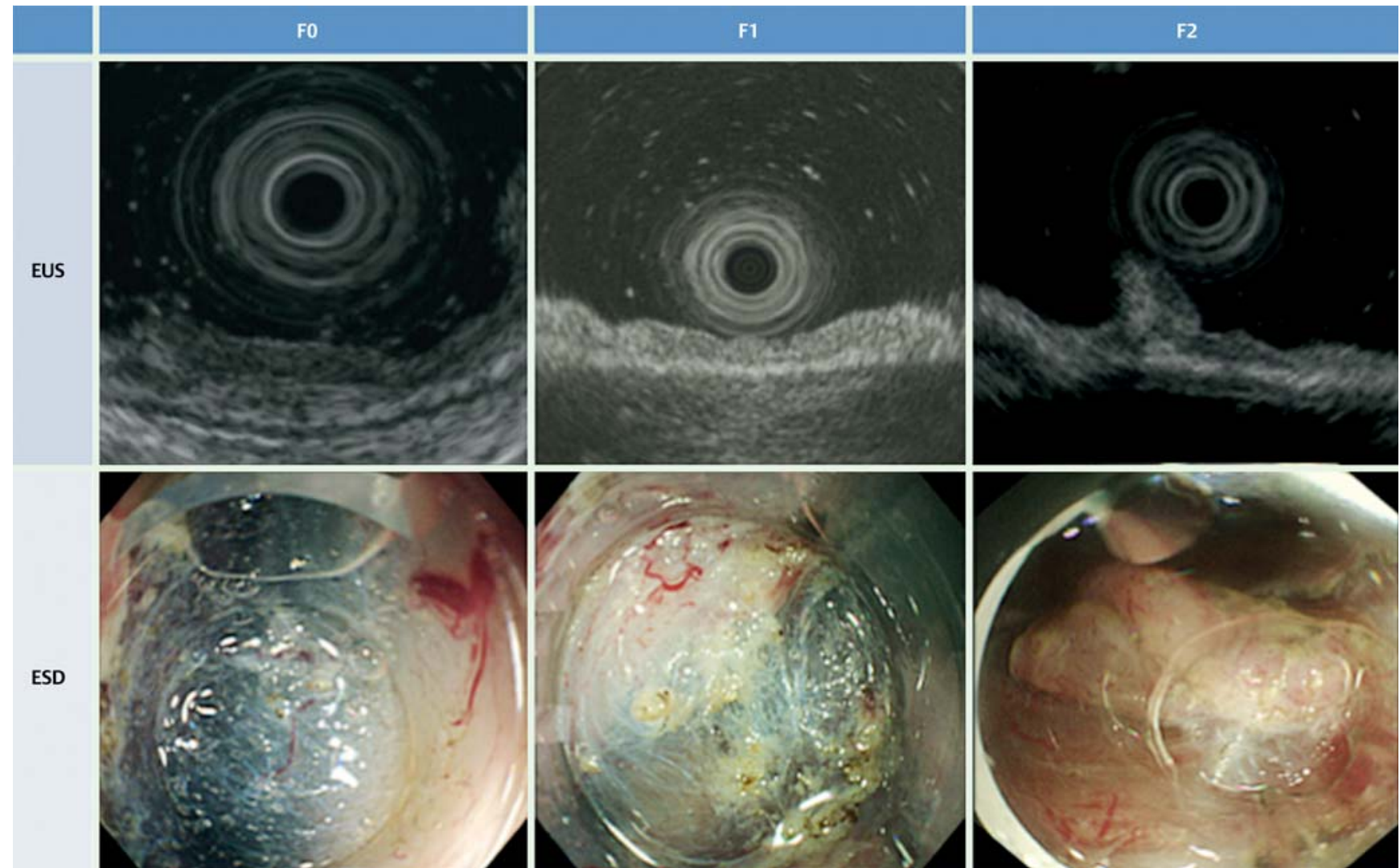

Fig. 1 Degree of fibrosis of submucosal layers in colorectal tumors. EUSF0: no fibrosis, where the width and brightness of the submucosal layer were constant compared to surrounding regions; EUS-F1: mild fibrosis, where the submucosal layer demonstrated local brightness compared to surrounding regions, or showed a slightly thickened submucosal layer; EUSF2: severe fibrosis, where the submucosal layer had high brightness com- pared to surrounding regions or showed a regionally thickened submucosal layer. ESD-F0: no fibrosis, which manifested as a blue transparent layer; ESDF1: mild fibrosis, which appeared as a white web-like structure in the blue submucosal layer; ESD-F2: severe fibrosis, which appeared as a white muscular structure without a blue transparent layer in the submucosal layer.
Gs) or non-granular type (LST-NGs). LST-Gs were further subdivided into homogenous-type and nodular-mixed-type tumors, and LST-NGs into flat-elevated-type and pseudo-depressed-type tumors. All patients underwent curative treatment by ESD or surgery, and all lesions were examined histopathologically. All patients provided their written, informed consent before ESD. This study was approved by the ethics committees of Kagoshima University Medical and Dental Hospital.

\section{EUS procedure}

After routine bowel preparation with a polyethylene-glycol solution, nonaerated water was injected and EUS was performed with a miniature probe set at $20 \mathrm{MHz}$ (Olympus, Tokyo, Japan). The colorectal wall was assessed based on the standard five-layer sonographic structure $[18,19]$. On the EUS image, the mucosal (M) layer is visualized as a combination of the first and second hypoechoic layers, and the submucosal (SM) layer corresponds to the third hyperechoic layer. The layer of the muscularis propria (MP) is visualized as the fourth hypoechoic layer, while the fifth hyperechoic layer is the serosa and includes the subserosa.

\section{ESD procedure}

ESD was performed in all patients using PCF-260AZI or Q260J (Olympus) endoscopes with dual knife or hook knife (Olympus). The submucosal injection solution contained $0.05 \%$ epinephrine and $0.1 \%$ indigo carmine in $10 \%$ glycerin and $5 \%$ fructose (Glyceol $^{\circledR}$; Chugai Pharmaceutical, Tokyo, Japan) or sodium hyaluro- nate (MucoUP ${ }^{\circledR}$, Johnson and Johnson, New Brunswick, NJ, United States) $[20,21]$.

Submucosal fibrosis assessment by EUS and ESD findings Fibrosis assessed by EUS was classified as follows ( 0 Fig. 1): no fibrosis, or EUS-F0 (submucosal layer's width and brightness were constant compared to surrounding regions); mild fibrosis, or EUS-F1 (submucosal layer was locally bright compared to surrounding regions or was mildly thickened); and severe fibrosis, or EUS-F2 (submucosal layer had high brightness compared to surrounding regions or was regionally thickened).

On histological analysis, the degree of submucosal fibrosis was classified into three grades based on the appearance of the layers following submucosal injection with the sodium hyaluronate and indigo carmine solution: ESD-F0, no fibrosis, which manifested as a blue transparent layer; ESD-F1, mild fibrosis, which appeared as a white, web-like structure in the blue submucosal layer; and ESD-F2, severe fibrosis, which appeared as a white muscular structure without a blue transparent layer [6] ( $\bullet$ Fig. 1). Two expert endoscopists diagnosed the severity of fibrosis estimated by EUS and the fibrosis observed during ESD by reviewing the still images. They were blinded to patients' identification information. 
Table 1 Patient characteristics $(n=58)$.

\begin{tabular}{|l|l|}
\hline Gender: male/female & $\mathbf{3 1 / 2 7}$ \\
\hline Mean age: years (range) & $70.6(43-88)$ \\
\hline Locations: C/A/T/D/S/R & $7 / 9 / 14 / 2 / 14 / 12$ \\
\hline Pathological findings: & \\
\hline Adenoma/adenocarcinoma & $30 / 28$ \\
\hline Histologic depth M/SM-s/SM-d/MP & $49 / 8 / 0 / 1$ \\
\hline Tumor size: mm (range) & $32.5(10-75)$ \\
\hline LST-G-H/G-M/NG-F/NG-PD & $6 / 26 / 8 / 18$ \\
\hline Fibrosis diagnosed by EUS (not evaluable/F0/F1/F2) & $22 / 24 / 5 / 7$ \\
\hline Fibrosis diagnosed by ESD (FO/F1/F2) & $30 / 19 / 9$ \\
\hline
\end{tabular}

Values are means \pm SD.

$\mathrm{C}$, cecum; A, ascending colon; T, transverse colon; $\mathrm{D}$, descending colon; S, sigmoid colon; R, rectum; M: mucosa; SM-s: slight invasion of the submucosa (submucosal invasion $<1000 \mu \mathrm{m}$ ); SM-d: deep invasion of the submucosa (submucosal invasion $>1000 \mu \mathrm{m})$; MP: muscularis propria. LST-G-H, laterally spreading tumor, granular, homogenous-type; LST-G-M, laterally spreading tumor, granular, nodular-mixed-type; LST-NG-F, laterally spreading tumor, non-granular, flat-elevated-type; LST-NG-PD, laterally spreading tumor, non-granular, pseudo-depressed-type.

\section{Statistical analysis}

Statistical evaluations were performed with the Mann-Whitney $U$-test and chi-squared test, using SPSS ver.15 (SPSS Inc., Chicago, IL, United States). The significance level was set at $P<0.05$.

\section{Results}

$\nabla$

\section{Patient characteristics}

The attributes of the 58 patients are shown in Table 1; $53 \%$ were male, and the mean age was $70.6 \pm 9.0$ years (range $43-$ 88). All patients underwent endoscopic resection by ESD. All cases were completely resected apart from one; in this instance, the ESD procedure was interrupted owing to severe submucosal fibrosis and additional surgical resection was therefore performed. There was no local recurrence in any cases. The average tumor size was $32.5 \pm 17.8 \mathrm{~mm}$. Twenty-eight cases were early CRC and 30 cases were high grade adenoma. LSTs were macroscopically classified as two subtypes: LST-Gs (32 cases) or LST-NGs (26 cases). These were further subdivided as follows: LST-Gs homogenous-type and nodular-mixed-type comprised 6 and 26 cases, respectively, and LST-NGs flat-elevated-type and pseudodepressed-type comprised 8 and 18 cases, respectively.

\section{Submucosal fibrosis findings by EUS}

EUS was used to evaluate 58 colon LSTs before colorectal ESD. Clear and analyzable scanned images were obtained from 36 of

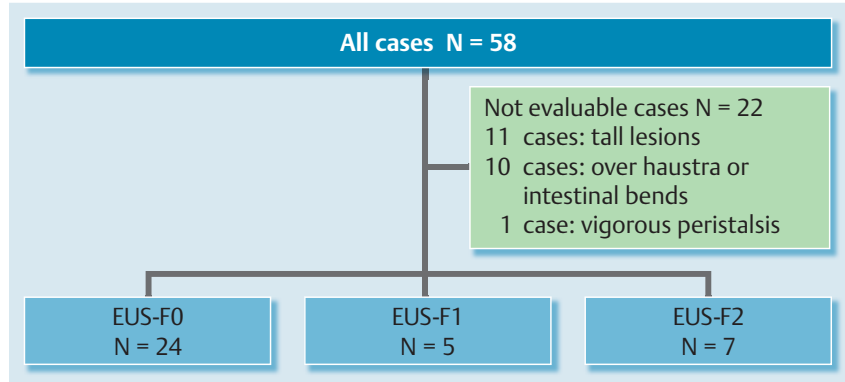

Fig. 2 Summary of all cases diagnosed by EUS.

58 LSTs. The remaining 22 cases were not evaluable by preoperative EUS for the following reasons: deep echo attenuation of tall lesions in 11 cases, tumor location (over haustra or intestinal bends) in 10 cases, and vigorous peristalsis in 1 case. EUS-F0 and -F1 were diagnosed in 24 and 5 cases, respectively, and EUS-F2 was diagnosed in 7 cases ( $\bullet$ Fig. 2 ).

\section{Submucosal fibrosis findings by ESD}

We defined two groups based on ESD findings: the first (ESD-F0/ F1) had no or mild fibrosis, and the second (ESD-F2) had severe fibrosis. The submucosal fibrosis assessed during ESD led to the diagnosis of 49 (30/19) cases as ESD-F0/F1 and nine as ESD-F2. There was no significant difference in gender, mean age, lesion location, or pathological findings between the ESD-F0/F1 and ESD-F2 groups ( $\bullet$ Table 2 ). Though there was no significant difference in procedure time (ESD-F0/F1: $110 \pm 65 \mathrm{~min}$; ESD-F2: $138 \pm 60 \mathrm{~min})$ between the two groups $(P=0.26)$, the mean tumor size in the ESD-F0/F1 group was significantly larger than that in ESD-F2 group (F0/F1: $34.9 \pm 18.3 \mathrm{~mm}$; F2: $20.1 \pm 6.6 \mathrm{~mm} ; \quad P=$ 0.002 ). There was also a significant difference between the two groups in procedure time per unit area (procedure time [min]/ tumor size $[\mathrm{mm}])(\mathrm{ESD}-\mathrm{F0} / \mathrm{F} 1: 3.3 \pm 1.6$; ESD-F2: $7.3 \pm 2.8)(P<$ 0.001). Perforation occurred in only one case in the ESD-F2 group, compared with none in the ESD-F0/F1 group.Thus, complication rates were higher in the presence of more severe fibrosis.

\section{Diagnostic accuracy of submucosal fibrosis}

Twenty-two lesions were difficult to evaluate using preoperative EUS. Of the remaining 36 that were readily evaluable, EUS predicted that 29 lesions would have no or mild fibrosis (EUS-F0/F1) and seven would have severe fibrosis (EUS-F2), yielding a sensitivity and specificity for fibrosis severity prediction of $77.8 \%$ (7/9) and $57.1 \%$ (28/49), respectively. Although the overall sensitivity

\begin{tabular}{|c|c|c|c|}
\hline & \multicolumn{2}{|c|}{ Degree of fibrosis determined by ESD } & \multirow[t]{2}{*}{$P$ value } \\
\hline & F0/F1 & F2 & \\
\hline Gender: male/female & $25 / 24$ & $6 / 3$ & 0.95 \\
\hline Mean age: years (range) & $70.8(43-88)$ & $69.8(58-79)$ & 0.61 \\
\hline Location: $C / A / T / D / S / R$ & $5 / 7 / 12 / 2 / 12 / 11$ & $2 / 2 / 2 / 0 / 2 / 1$ & 0.53 \\
\hline Pathology: adenoma/carcinoma & $25 / 24$ & $5 / 4$ & 0.49 \\
\hline Tumor size: mm (range) & $34.9(13-75)$ & $20.1(10-30)$ & 0.002 \\
\hline Procedure duration: min & $110.0 \pm 65.3$ & $137.7 \pm 60.3$ & 0.26 \\
\hline Procedure time per unit area ${ }^{1}$ & $3.3 \pm 1.6$ & $7.3 \pm 2.8$ & $<0.001$ \\
\hline Perforation & 0 & 1 & - \\
\hline
\end{tabular}

Table 2 Comparisons between F0/F1 and F2 groups $(n=58)$.

Values are means \pm SD.

C, cecum; A, ascending colon; $\mathrm{T}$, transverse colon; $\mathrm{D}$, descending colon; $\mathrm{S}$, sigmoid colon; $\mathrm{R}$, rectum.

1 Procedure time per unit area: procedure time $(\mathrm{min}) /$ tumor size $(\mathrm{mm})$. 
and specificity for fibrosis severity prediction were $77.8 \%$ and $57.1 \%$, respectively, for just the 36 LSTs in which we could obtain clear scanned images, the sensitivity, specificity, positive predictive value, negative predictive value, and accuracy in discriminating $\mathrm{F} 0 / \mathrm{F} 1$ and $\mathrm{F} 2$ were $87.5 \%, 100 \%, 100 \%, 96.6 \%$, and $97.2 \%$, respectively ( Table $\mathbf{3})$.

\section{Discussion}

$\nabla$

We demonstrated the utility of EUS for predicting submucosal fibrosis of CRC lesions before ESD for colorectal LST. It is important to correctly determine these parameters before ESD because they influence the surgical treatment performed. The sensitivity and specificity for fibrosis severity prediction were $77.8 \%$ and $57.1 \%$, respectively, which were reasonable. However, in the 36 cases in which we could obtain clear scanned images, the sensitivity, specificity, and accuracy for prediction of fibrosis severity were excellent. One case was predicted by EUS to have no fibrosis (EUS-F0), but was eventually diagnosed with severe fibrosis (ESD-F2) based on ESD findings. This case had tumor invasion into the submucosa. We misjudged the degree of submucosal fi-
Table 3 Diagnostic accuracy of fibrosis $(n=36)$.

\begin{tabular}{|llc} 
EUS diagnosis & \multicolumn{2}{l}{ Degree of fibrosis determined during ESD } \\
\cline { 2 - 3 } & Severe & None/Mild \\
\hline F2 & 7 & 0 \\
F0/F1 & 1 & 28 \\
\hline
\end{tabular}

Sensitivity, $87.5 \%$ (7/8); specificity, $100 \%$ (28/28); positive predictive value, $100 \%$ (7/7); negative predictive value, 96.6\% (28/29); accuracy, $97.2 \%$ (35/36).

brosis, because the fibrosis area in the submucosa might be narrow ( $\bullet$ Fig 3).

In addition, the diagnosis of gastrointestinal tumors is based on endoscopic and histopathologic findings, and EUS has recently been shown to be the most accurate method for predicting the depth of gastric and colorectal neoplasms in order to determine clinical staging for the most appropriate management plan [22, 23]. In this study, we used EUS to successfully determine the depth of 35 of 36 LSTs for which clear scanned images could be obtained, located in the mucosa (M) or with slight invasion of the submucosa (SM-s), with an accuracy of $97.2 \%$. Although this
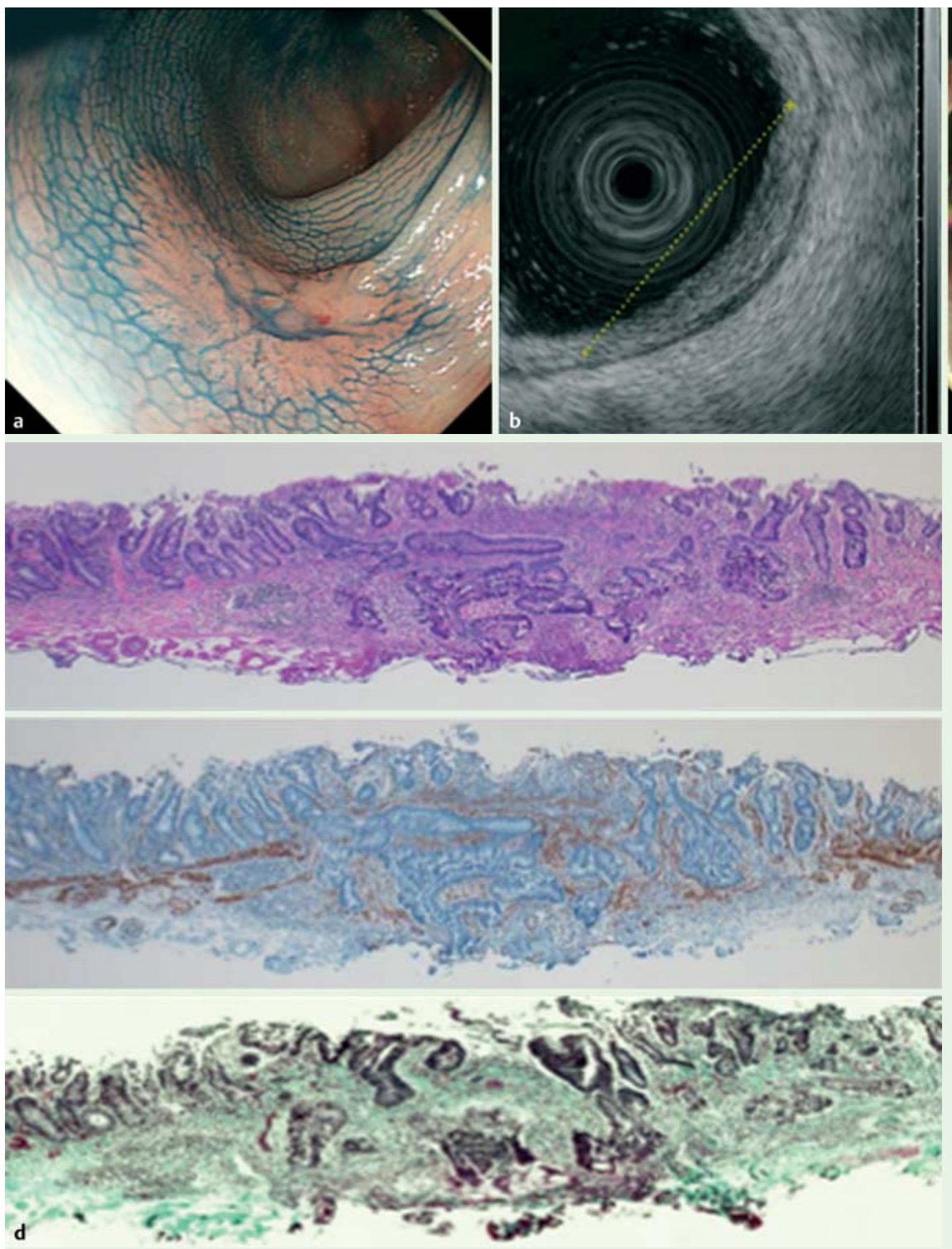

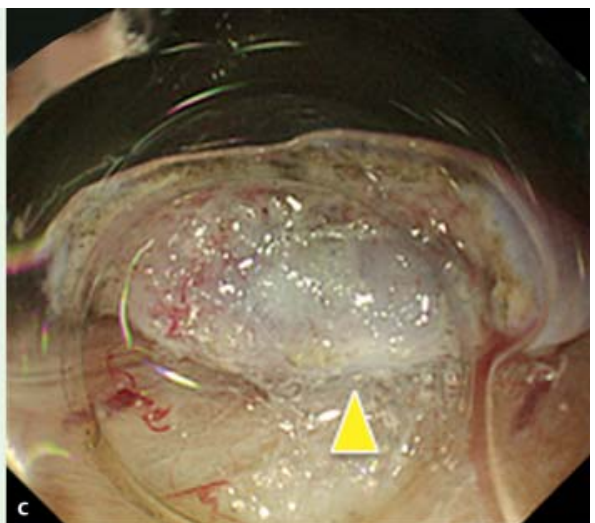

Fig. 3 A case with severe fibrosis despite diagnosis as EUS-FO before ESD. a Conventional colonoscopy image stained with indigo-carmine. $\mathbf{b}$ EUS shows normal submucosal layer width and no hyperechoic area in the submucosa. c Severe fibrosis visualized during ESD (arrowhead). d Microscopic cross-sectional view demonstrates tumor invasion into the submucosa on hematoxylin and eosin staining (upper panel), and smooth muscle antigen staining (middle panel), as well as submucosal fibrosis on hematoxylin and eosin staining, and Masson trichrome staining (lower panel). 
was a small-scale study, the utility of EUS for assessing CRC invasion confirmed the findings of previous studies [9,15-17].

ESD is a more effective procedure for treating early CRC than conventional EMR. In addition, ESD results in a higher en bloc resection rate and is less invasive than surgery [24]. However, the technical difficulty of colorectal ESD is higher for LST than for conventional EMR, as shown by the longer procedure time and higher perforation rate. According to previous reports, perforation rates range from 1.4 to $5.5 \%$ [25-27]. In addition, because the colorectal wall is thin compared with the stomach or esophagus, intestinal submucosal fibrosis reduces the safety of colorectal ESD. This procedure should therefore be performed by clinicians with significant experience with gastrointestinal ESD. Severe fibrosis is associated with a much longer procedure time and higher risk of complications such as intestinal perforation [6]. Furthermore, the accurate prediction of fibrosis is very important before colorectal ESD; it is also necessary to consider the patient's background and the skill of the operator. Together, these factors can be used to predict the technical difficulty of a given colorectal ESD procedure, allowing more experienced clinicians to be assigned to more difficult cases and thus leading to a reduction in complications.

EUS with the miniature probe set at $20 \mathrm{MHz}$ has been widely used for scanning superficial gastrointestinal tumors. It is likely, therefore, that EUS will be useful for predicting submucosal fibrosis in superficial colonic lesions such as LST before ESD. However, it was difficult to obtain clear and analyzable scanned images by preoperative EUS at $20 \mathrm{MHz}$ owing to the deep echo attenuation of tall or protruding lesions. The accuracy of diagnosing submucosal fibrosis of tall lesions was low at our facility (data not shown). The reliability of diagnostic images of the submucosal layer of tall lesions without marked echo attenuation may be increased in the future by performing preoperative EUS using a radial echoendoscope (such as GF-UE260 or UM2000).

Although EUS is a feasible method for assessing the degree of submucosal fibrosis of LSTs before colorectal ESD, this study has an important limitation. This was a retrospective, single-center study, with the gold standard being fibrosis assessed by ESD results, not by pathological findings. We should conduct a prospective, multi-center study to assess submucosal fibrosis before colorectal ESD in the near future.

In conclusion, EUS can predict the degree of submucosal fibrosis of CRC lesions before colorectal ESD, and may allow clinicians to stratify patients based on the technical difficulty anticipated for their ESD procedure.

\section{Competing interests: None}

\section{References}

1 Ferlay J, Shin HR, Bray F et al. Estimates of worldwide burden of cancer in 2008: GLOBOCAN 2008. Int J Cancer 2010; 127: 2893-2917

2 Fujishiro M. Perspective on the practical indications of endoscopic submucosal dissection of gastro intestinal neoplasm. World J Gastroenterol 2008; 14: 4289-4295

3 Kakushima N, Fujishiro M. Endoscopic submucosal dissection for gastrointestinal neoplasms. World J Gastroenterol 2008; 14: 2962-2967

4 Terasaki M, Tanaka S, Oka S et al. Clinical outcomes of endoscopic submucosal dissection and endoscopic mucosal resection for laterally spreading tumors larger than $20 \mathrm{~mm}$. J Gastroenterol Hepatol 2012; 27: $734-740$
5 Hizawa $K$, Iwai $K$, Esaki $M$ et al. Is endoscopic ultrasonography indispensable in assessing the appropriateness of endoscopic resection for gastric cancer? Endoscopy 2002; 34: 973-978

6 Matsumoto A, Tanaka S, Oba A et al. Outcome of endoscopic submucosal dissection for colorectal tumors accompanied by fibrosis. Scand J Gastroenterol 2010; 45: 1329-1337

7 Isomoto $\mathrm{H}$, Nishiyama $\mathrm{H}$, Yamaguchi $\mathrm{N}$ et al. Clinicopathological factors associated with clinical outcomes of endoscopic submucosal dissection for colorectal epithelial neoplasms. Endoscopy 2009; 41: 679-683

8 Nishiyama H, Isomoto $H$, Yamaguchi $N$ et al. Endoscopic submucosal dissection for colorectal epithelial neoplasms. Dis Colon Rectum 2010; 53: $161-168$

9 Saitoh Y, Obara T, Einami K et al. Efficacy of high-frequency ultrasound probes for the preoperative staging of invasion depth in flat and depressed colorectal tumors. Gastrointest Endosc 1996; 44: 34-39

10 Kobayashi K, Kida M, Katsumata $T$ et al. Clinical role of endoscopic ultrasonography for the diagnosis of early colorectal cancer and selecting the treatment procedure. Dig Endosc 2003; 15: 298-305

11 Akasu T, Kondo H, Moriya $Y$ et al. Endorectal ultrasonography and treatment of early stage rectal cancer. World J Surg 2000; 24: 1061 1068

12 Hizawa K, Suekane H, Aoyagi K et al. Use of endosonographic evaluation of colorectal tumor depth in determining the appropriateness of endoscopic mucosal resection. Am J Gastroenterol 1996; 91: 768 - 771

13 Cho E, Nakajima M, Yasuda K et al. Endoscopic ultrasonography in the diagnosis of colorectal cancer invasion. Gastrointest Endosc 1993; 39: $521-527$

14 Norton SA, Thomas MG. Staging of rectosigmoid neoplasia with colonoscopic endoluminal ultrasonography. Br J Surg 1999; 86: 942 - 946

15 Konishi $K$, Akita $Y$, Kaneko $K$ et al. Evaluation of endoscopic ultrasonography in colorectal villous lesions. Int J Colorectal Dis 2003; 18: 1924

16 Tsuruta $\mathrm{O}$, Kawano $\mathrm{H}$, Fujita $\mathrm{M}$ et al. Usefulness of the high-frequency ultrasound probe in pretherapeutic staging of superficial-type colorectal tumors. Int J Oncol 1998; 13: 677-684

17 Ohashi S, Segawa K, Okamura S et al. The utility of endoscopic ultrasonography and endoscopy in the endoscopic mucosal resection of early gastric cancer. Gut 1999; 45: 599-604

18 Yanai H, Matsumoto Y, Harada T et al. Endoscopic ultrasonography and endoscopy for staging depth of invasion in early gastric cancer: a pilot study. Gastrointest Endosc 1997; 46: $212-216$

19 Kimmey MB, Martin RW, Haggitt RC et al. Histologic correlates of gastrointestinal ultrasound images. Gastroenterology 1989; 96: 433-441

20 Yamamoto H, Sekine Y, Higashizawa T et al. Successful en bloc resection of a large superficial gastric cancer by using sodium hyaluronate and electrocautery incision forceps. Gastrointest Endosc 2001; 54: 629632

21 Yamamoto $H$, Yube T, Isoda $N$ et al. A novel method of endoscopic mucosal resection using sodium hyaluronate. Gastrointest Endosc 1999; 50: $251-256$

22 Akahoshi K, Chijiwa Y, Hamada S et al. Pretreatment staging of endoscopically early gastric cancer with a $15-\mathrm{MHz}$ ultrasound catheter probe. Gastrointest Endosc 1998; 48: 470-476

23 Hurlstone DP, Brown S, Cross SS et al. High magnification chromoscopic colonoscopy or high frequency $20 \mathrm{MHz}$ mini probe endoscopic ultrasound staging for early colorectal neoplasia: a comparative prospective analysis. Gut 2005; 54: 1585 - 1589

24 Saito $Y$, Ymada $M$, So $E$ et al. Colorectal endoscopic submucosal dissection: Technical advantages compared to endoscopic mucosal resection and minimally invasive surgery. Dig Endosc 2014; 26: 52 -61

25 Tanaka S, Haruma $K$, Oka S et al. Clinicopathologic features and endoscopic treatment of superficially spreading colorectal neoplasms larger than 20 mm. Gastrointest Endosc 2001; 54: 62 - 66

26 Fujishiro M, Yahagi N, Kakushima N et al. Outcomes of endoscopic submucosal dissection for colorectal epithelial neoplasms in 200 consecutive cases. Clin Gastroenterol Hepatol 2007; 5: 678-683

27 Tamegai Y, Saito Y, Masaki N et al. Endoscopic submucosal dissection: a safe technique for colorectal tumors. Endoscopy 2007; 39: 418-422 